\title{
IN FLIGHT DETECTION AND LOCALISATION OF SHORT CIRCUITS ON SOLAR GENERATORS
}

\author{
Henri Barde ${ }^{(1)}$, Emmanuelle Carla ${ }^{(2)}$, Nicolas Neugnot ${ }^{(2)}$, Etienne Rapp ${ }^{(3)}$ \\ (1) European Space Agency (ESA), henri.barde@esa.int \\ (2) Airbus DS France, 31 rue des Cosmonautes 31402 Toulouse France, Email:emmanuelle.carla@airbus.com \\ (3) CNES, 2 place Maurice Quentin 75039 Paris Cedex 01 France, Email:Etienne.Rapp@cnes.fr
}

\begin{abstract}
In the last decades a number of failures in which a solar cell is short-circuited to the panel substrate have been reported or suspected. In the meantime protective measures, like single string connection and use of bleed resistors in the grounding circuit have been implemented. However it is still not always clear whether such an anomaly occurred and the in-flight instrumentation is usually not sufficient to provide unambiguous evidence. This paper describes a simple way to identify both the occurrence of such a cell to substrate short circuit and the panel on which it is located.

The paper is then completed with the main results from the CNES study performed in 2012 to demonstrate the performance, analyse the reliability and assess the industrial feasibility of such a system.
\end{abstract}

\section{INTRODUCTION}

Detailed information on failure occurring in orbiting spacecraft is seldom released. However, when looking at the history of spacecraft anomalies, it appears that solar array failures represent a very significant fraction of the insurance claims [1]. Even if not all of them are of electrical nature (deployment failures are a significant contributor) it is known, even if not published, that cell to substrate short circuits are far from being uncommon. Experience also shows that identifying the exact cause of an anomaly and especially if the suspected short circuit is the result of an external cause, like a micro meteorite impact, or a consequence of another electrical event is challenging. The usual spacecraft instrumentation is designed to support nominal and anticipated contingency operations, but in general not to help a detailed investigation. The present paper is a contribution to this issue.

\section{DESCRIPTION OF THE PROBLEM}

The earlier designs of solar generators generally made use of a direct connection of the panels structure to the spacecraft electrical reference point, for the purpose of avoiding large differential voltages to build up. As shown in a simplified way in Figure 1, the consequence of this direct connection was that a short-circuit of a cell to the substrate could result into a loss of power, potentially significant if the cells were connected both in series and parallel (in a so-called matrix configuration). Some spacecraft built in the 1980s experienced a significant amount of such power reduction, which, in some cases, were synchronous of

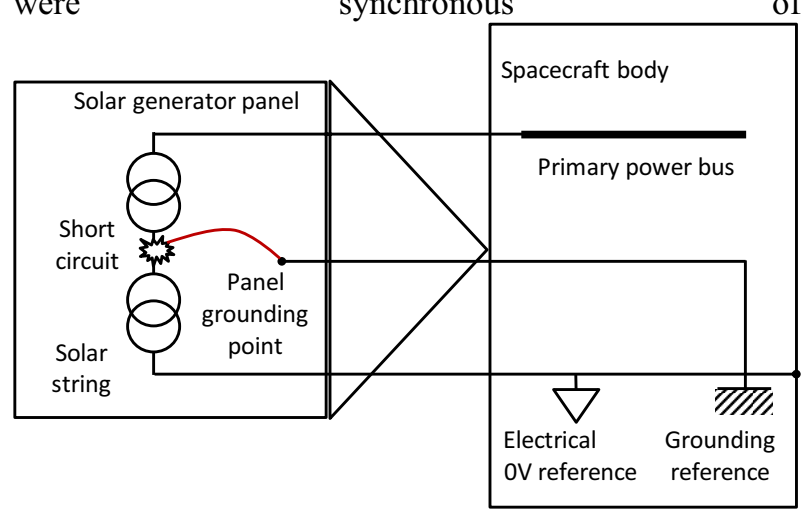

Figure 1: Direct electrical connection of solar array structure to spacecraft body

an attitude disturbance, suggesting that an impact could have been at the origin of this power loss. In other cases, this could also be interpreted as an ejection of mass, as the result of a component blow-up.

The relatively frequent occurrence of such phenomena led to the implementation of preventive measures, namely:

- The "single string" layout, where each string of series connected cells is paralleled to the other ones via a blocking diode, limiting any potential loss to this of the affected string;

- The electrical isolation of the structure panels between them and from the spacecraft electrical reference point;

- And the introduction of bleed resistors of a few 10 $\mathrm{k} \Omega$, to provide a path for charges to flow and so ensure a limited voltage gradient between both.

This is summarised in Figure 2.

With these protections, a cell to substrate short circuit occurring on a panel can only produce a negligible stray current and at least two such events have to occur on the same panel to induce the loss of one string.

However, even with these precautions taken, some failures are still reported and raise the question of the actual "chicken and egg" situation mentioned above: are impacts frequent, but not visible as long as they do not affect several times the same panel, or are the occasional disturbances seen on the attitude the result of energetic 
events generated on-board? Being able to detect such short circuits even when they do not produce observable effects would contribute to a better understanding of the problem.

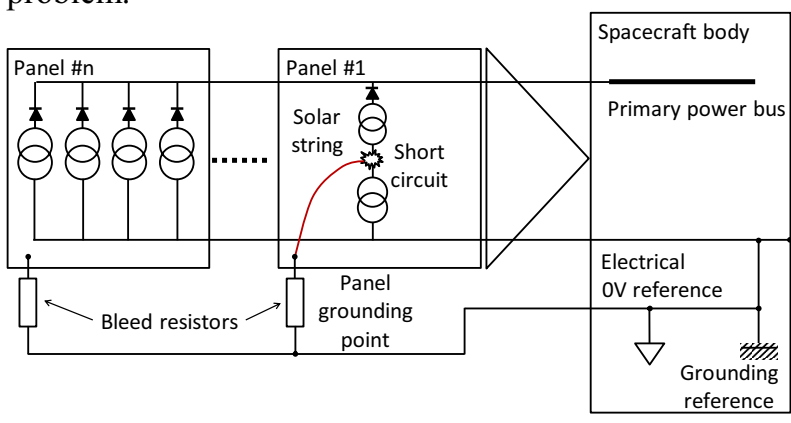

Figure 2: Protective measures against cell to substrate short circuit

\section{PROPOSED SOLUTION}

The first step is the detection of the short-circuit. To this end the network of bleed resistors usually in place can be slightly modified to provide this information, as shown in Figure 3, by the introduction of an additional sense resistor between the panels grounding line and the spacecraft electrical reference.

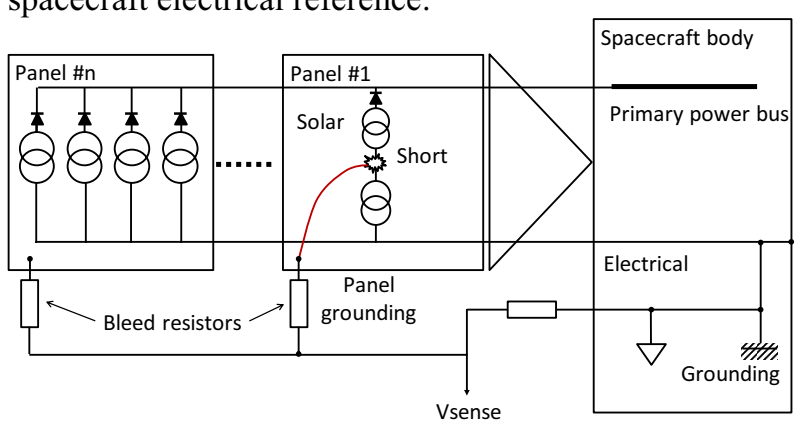

Figure 3: Protective measures against cell to substrate short circuit

In the absence of any short-circuit, the voltage Vsense across this resistor is essentially null, being the product of its resistance by the charge draining current, in the range of nanoamperes. Now, when a cell becomes shortcircuited to a panel, the voltage across the fraction of the string between the zero volt and this cell is applied to the resistive network made of the bleed resistors and of the sense resistor. Knowing their respective values allows to determine the voltage at the short circuit, i.e. the position of the failed cell inside its string.

The selection of the ohmic value of the sense resistor depends on the maximum voltage that can be reached by a string, the number of short-circuits that may exist and the input voltage range of the analogue telemetry. As an example, for a 3 panels array with an upper cell shorted on each, a maximum voltage of $100 \mathrm{~V}$ (beginning of life, cold conditions, full illumination) and a $5 \mathrm{~V}$ telemetry range, the sense resistor value should not be larger than $5 / 300$ of the common value of the bleed resistors. In this case a short-circuit on a single panel at half the string in normal operating conditions would be around a few $10 \%$ of the full range, which is acceptable.

More information can be extracted from this very simple circuit, though, considering that, in its practical implementation, the grounding network is redundant. By properly selecting the values of the prime and redundant resistors, different divider ratio can be set for each solar panel, making possible the identification of the panel on which the short circuit has occurred. This is illustrated in a simplified form by Figure 4, where a three panels array is considered.

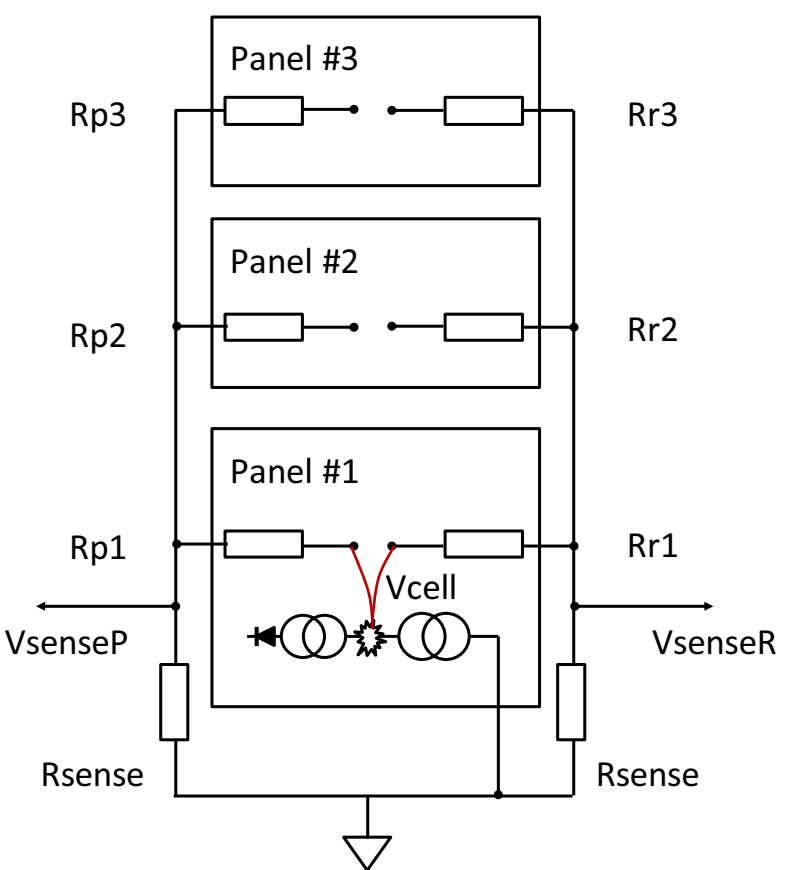

Figure 4: Use of both prime and redundant grounding networks for short circuit localisation

The ratio between VsenseP and VsenseR is given by:

$$
\frac{V \text { senseP }}{V \text { senseR }}=\frac{R 1+R \text { sense }}{R 2+R \text { sense }}
$$

where

$$
\begin{gathered}
R 1=\frac{R \cdot R r 1}{R p 1+R r 1+R} \\
\mathrm{R} 2=\operatorname{Rp} 1 \cdot \frac{\mathrm{R}}{\mathrm{Rp} 1+\operatorname{Rr} 1+\mathrm{R}}
\end{gathered}
$$

and

$$
R=\frac{(R p 2+R r 2) \cdot(R p 3+R r 3)}{R p 2+R r 2+R p 3+R r 3}
$$

The details of the quite simple calculation are provided in [2]. Therefore if all ratios on Vsense $P$ V sense $R=\frac{R 1+R \text { sense }}{R 2+\text { Rsense }}$ right side are different, which is easy to ensure by a 
proper selection of the bleed resistors values, then the identification of the panel is unambiguous. For instance a series of increasing ohmic values can be attributed to the prime side and the same series in opposite order on the redundant one.

At this stage the benefit is already significant, since the identification of the panel provides an indication of the distance from the spacecraft centre of mass at which the impact or the ejection occurred. In addition, in the relatively frequent situation where the strings are laid down parallel to the spacecraft body (as illustrated by Figure 5) the identification of the shorted cell together with the characteristics of the attitude disturbance allow to know on which side of the panel the event occurred. This is important in making the distinction between e.g. a micro-meteorite impinging on a cell or the ejection of a blown component from the rear side.

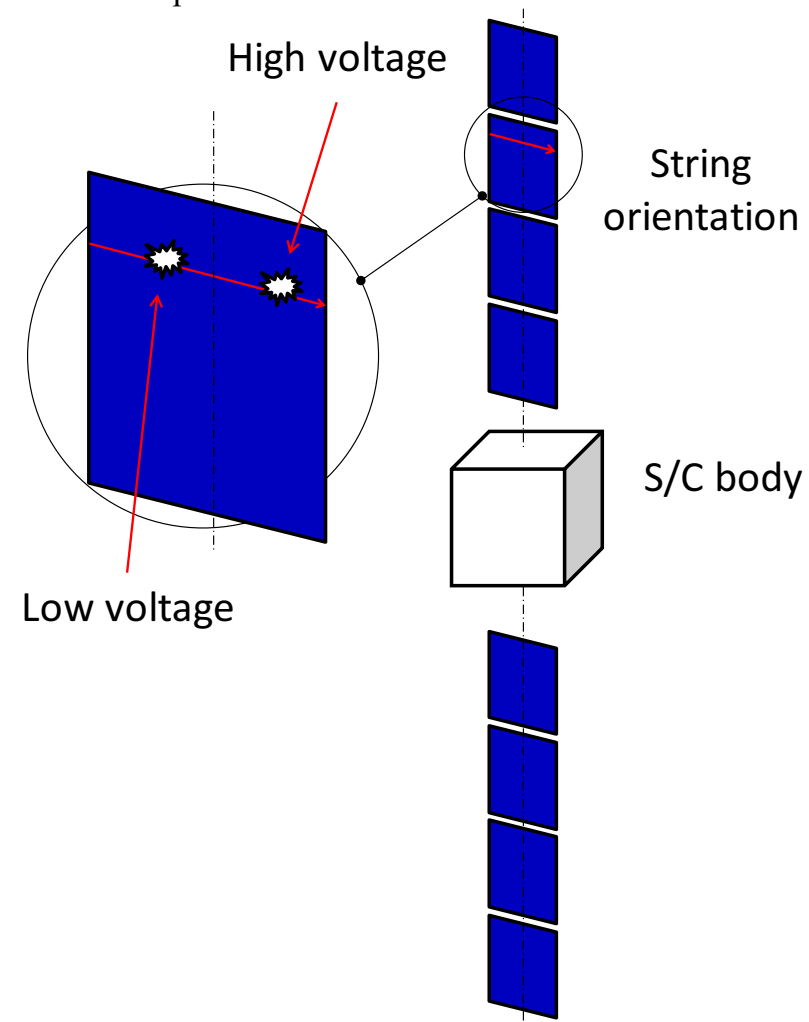

Figure 5: Knowledge of panel and rank in the string of short-circuited cell allows approximate location of the defect

\section{OTHER ASPECTS OF THE CIRCUIT}

\subsection{Measurement noise and filtering}

The normal operation of the solar array regulator generates an AC component of the current delivered by one or more sections of the solar array. A S3R [3], for instance, will produce a square wave, the current of the toggling section varying from its operating to shortcircuit value. The capacitive coupling that exists between the cells and the substrates means that this $\mathrm{AC}$ component will be picked up by the circuit described above. It is therefore advisable to filter out this noise, e.g. by connecting a capacitor in parallel to the sense resistor.

\subsection{Selection of bleed resistors}

Implementing resistors of different values on each panel has the drawback of introducing a slight complication in the design and procurement process. A way to reduce this - modest - added burden is to achieve the different ohmic values by adequate series/parallel arrangement of identical resistors. For instance, the circuit of Figure 4 could be made of resistors of $2 R, R$ and $R / 2$ value by using respectively two in series, a single one or two in parallel. The corresponding ratios between VsenseP and VsenseR would then be in the order of 4, 1 and 0.25 , values that can be easily discriminated. As a side positive - effect, having two different values for the same information allows to process the highest one to achieve the best resolution.

\subsection{Multiple events}

It is important to assess if, in case of multiple shortcircuits, on one or several panels, it is still possible to identify, for each of them, both the applied voltage and the panel location.

This is rather straightforward for multiple events on the same panel: whatever the characteristics of the source voltage, resulting from the combination of the various strings involved, the ratio between the two sensed voltages will stay the same. It may be more difficult to understand the exact configuration but, at least, further events would be detected, as long as they modify the source voltage.

In case a second event occurs on a panel different from the one hosting the initial short-circuit, the situation is more complex: indeed different combinations of panel and applied voltage may produce the same VsenseP/VsenseR ratio. This is illustrated by Figure 6 where a second failure, generating a panel voltage between zero and twice the first panel one, is applied either to panel 2 or panel 3. One can see that a range of ratio values are common to both panels. In this case, the initial ratio, after the first failure, is close to 0.26 , i.e. a short-circuit close to the negative side of a string would probably not be detected. In most of the cases, though, a new short would modify the sensed voltages and, for a range of values, would allow identification of the newly affected panel. In the example shown, any value of the ratio above 0.64 would be indicative of a short circuit on panel \#3.

In summary:

- Any short-circuit occurring on a first panel would be detected; the panel and applied voltage would be identified;

- Any further short-circuit on the same panel would be detected but the exact event would be difficult to characterise;

- Any short-circuit occurring on another panel would 
be detected, provided that it concerns a sizeable fraction of the string; the panel identification may or may not be possible, depending of the applied voltage.

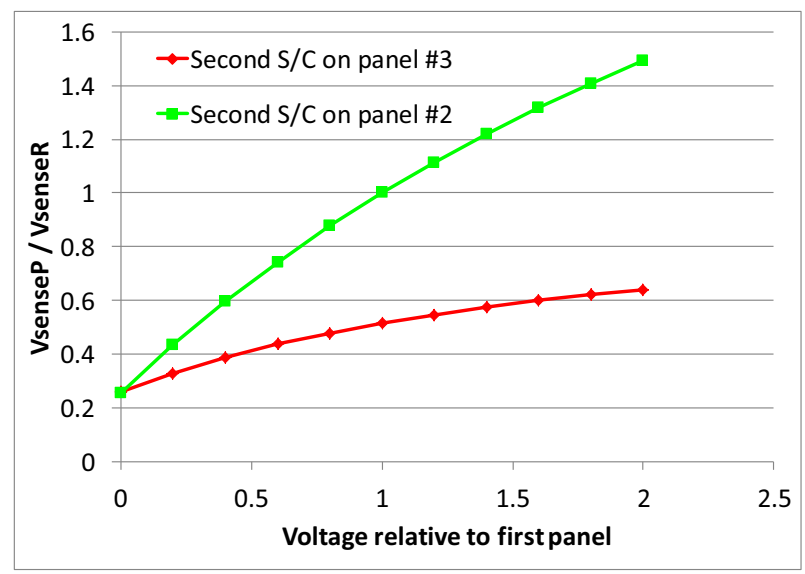

Figure 6: VsenseP/VsenseR ratio as a function of the second short-circuit voltage, the panel as a parameter

\section{RELIABILITY ANALYSIS}

The objective of reliability analysis is to consolidate the previous design but also to check that the operation of the satellite is not degraded even in the event of a failure. For that, several analyses have been performed in the frame of the CNES study and are presented hereafter:

- ESD: The ESD analysis allowed to check that the detection system is immune to the phenomena of electrostatic discharge on the one hand and that it is not likely to trigger an electrostatic discharge on the other hand, even in the event of a failure. This is justified by the fact that there are no metallic parts floating since they are always connected through a bleed resistance to the spacecraft structure, and because no new materials are included (same harness and same bleed resistors used).

-FMECA: Failure Mode, Effects and Criticality Analysis proved that no failure can degrade the operation of the satellite. In fact, in event of failure only the localization and detection function can be lost but no propagation to the satellite.

- Stress analysis: During a failure, system components will not exceed their nominal operating conditions.

\section{INDUSTRIALIZATION PLAN}

The conclusion of the CNES study shows that for a solar array configuration that is to say between 3 and 5 panels, it is possible to detect the defective panel when the default voltage exceeds $10 \mathrm{~V}$ for a $50 \mathrm{~V}$ bus and $20 \mathrm{~V}$ for a $100 \mathrm{~V}$ bus, considering the worst case tolerances. Moreover, it is possible to discriminate the cell where the default occurs with a precision of $+/-1$ cell for a $50 \mathrm{~V}$ bus and $+/-2$ cells for a $100 \mathrm{~V}$ bus. Noise due to power supply regulator switching can add temporary uncertainties (few seconds) on the localization performances approximately of $+/-1$ cell. This is due to a power variation on the bus (and therefore a modification of duty cycle or commutation from section).

It seems definitely a very interesting idea that should be applied without much effort. To do so, an industrialization plan has been defined for Airbus DS telecom platform Eurostar 3000 (E3000).

The list of materials has been gathered and all the components required for this application are already qualified and used on the current platform. No additional and expensive qualification campaigns are therefore required.

The location of the additional resistors, the type of wire and the connector including the filter have been analysed without any showstopper identified so far.

The industrialization on Eurostar 3000 platform is therefore demonstrated and currently waiting for a potential customer willing to apply it. Other programs can also take advantage of such technology like the Astrobus platform, Metop-NG...

\section{CONCLUSION}

The circuit described in this paper allows, at a very moderate expense, to detect almost all short-circuits that could occur between solar cells and substrate in a solar generator and in many cases to identify the affected panel and the voltage at the short-circuit.

Its implementation on spacecraft would allow to get a very valuable statistic of such events and, in some specific failure cases, to bring an important contribution to the understanding of the causes.

\section{REFERENCES}

1. Rodiek, J.A. \& Brandhorst H.W.Jr. (2008) Solar array reliability in satellite operations, Photovoltaic Specialists Conference, 2008. PVSC '08. 33rd IEEE.

2. Carla E., Barde H., System and method for detecting and locating an insulation flaw in a solar generator on a space vehicle, US patent US 2014/0232416

3. Weinberg A.H., O'Sullivan D.M., Limit cycling regulator apparatus for plural parallel power sources, US patent US4186336 A 